\title{
Music Modulates the Strength of Vection
}

\author{
Takeharu Seno ${ }^{1,2,3}$ \\ ${ }^{1}$ Faculty of Design, Kyushu University, Fukuoka, Japan \\ ${ }^{2}$ Institute for Advanced Study, Kyushu University, Fukuoka, Japan \\ ${ }^{3}$ Research Center for Applied Perceptual Science, Kyushu University, Fukuoka, Japan \\ Email: seno@design.kyushu-u.ac.jp
}

Received April 20 $0^{\text {th }}$, 2013; revised May 23 ${ }^{\text {rd }}$, 2013; accepted June $20^{\text {th }}, 2013$

\begin{abstract}
Copyright (c) 2013 Takeharu Seno. This is an open access article distributed under the Creative Commons Attribution License, which permits unrestricted use, distribution, and reproduction in any medium, provided the original work is properly cited.
\end{abstract}

\begin{abstract}
We presented four types of music (two fast tempo and two slow tempo types) during illusory self-motion perception (vection). Vection was induced by expansional dots (optic flow), and participants estimated its strength via magnitude estimation and by pressing a button. Our purpose of this study is to examine whether music alter vection strength. Results showed that vection was facilitated by two fast tempo types and one slow tempo type of music in lieu of a no-music condition. We speculated that fast tempo, active music might induce higher arousal levels in participants compared with a no-music condition, and that higher arousal levels might induce stronger vection. We speculated that this auditory method of modulating vection strength can be utilized in the virtual reality environment.
\end{abstract}

Keywords: Vection; Music; Sound; Facilitation

\section{Introduction}

Music is known to influence human behavior (Review in Miell et al., 2005). For instance, when listening to music with a fast tempo, supermarket shoppers have been found to move more quickly around a store, restaurant patrons eat more quickly, and drinks in pubs are consumed more quickly, than when listening to music with a slow tempo (Milliman, 1982, 1986; Herrington \& Capella, 1996). Additionally, listening to music with a fast tempo increases the rate and precision of mathematical computations in stock-market environments (Mayfield \& Moss, 1989), and of self-paced line drawings (Nittono et al., 2000).

Recent studies have revealed a relationship between music and self-motion, i.e. driving. Spinney (1997) found that music exposure during driving facilitated avoidance of driving hazards and increased performance, such as reaction times. In addition, drivers characterized scenic countryside landscapes as having more power when listening to music with a fast tempo (Iwamiya, 1997). Another study found that music tempo consistently affected simulated driving speed, perceived speed, and the frequency of virtual traffic violations (Brodsky, 2002).

In this study, we focused on the effect of music on illusory self-motion perception, vection. Vection refers to the phenomenon in which a stationary observer experiences compelling illusory self-motion when exposed to a large visual field of optic flow (e.g. Seno et al., 2013).

Music has been shown to have the ability to alter human arousal levels (Gabrielsson, 2001). Specifically, fast tempo music has been found to induce higher arousal levels while slow tempo music has been found to induce lower arousal levels (Husain et al., 2002). In our recent study, we found that vection could also alter human arousal levels; stronger vection induced higher arousal levels (Ihaya et al., submitted). We hy- pothesized that the opposite was also possible, i.e., that higher arousal levels could induce stronger vection. We further hypothesized that when arousal levels were increased by fast tempo music, these higher arousal levels might induce stronger vection. In this way, we predicted that fast tempo music could facilitate vection.

\section{Method}

\section{Apparatus}

Stimuli were generated and controlled by a computer (MB 543J/A, Apple) and presented on a plasma display (3D Viera, 50 -inch, Panasonic, with $1024 \times 768$ pixel resolution at a $60 \mathrm{~Hz}$ refresh rate). The experiment was conducted in a dark chamber.

\section{Participants}

Fourteen naïve volunteers participated in this experiment. Participants were graduate or undergraduate students. All participants reported normal vision and no history of vestibular system diseases. None of them was aware of the purpose of the experiment.

\section{Stimuli}

Vection was induced in fourteen stationary observers by presenting expanding optic flow. Optic flow displays $\left(72^{\circ} \times 57^{\circ}\right.$; presented for $30 \mathrm{~s}$ ) consisted of 16,000 randomly positioned dots (Seno et al., 2010) with global dot motion to simulate forward self-motion $(16 \mathrm{~m} / \mathrm{s})$.

\section{Procedure}

Participants were asked to press a button when they per- 
ceived forward self-motion, and keep the button depressed for the duration of self-motion. After each trial, the participants rated subjective vection strength using a 101-point rating scale ranging from 0 (no vection) to 100 (very strong vection).

There were five music conditions: two fast, two slow, and a no-music condition. These conditions were conducted in separate sessions. Three trials were conducted for the with-music conditions and four trials for the without-music condition. For the two fast music conditions, we used "Bakushou-sengen" (the theme of a Japanese Pro-wrestler) and the theme music from the movie "Back to the Future", and for two slow music conditions, we used Pachelbel's “Kanon-D-dur” and Debussy's "Moon Light". The three trials were conducted successively within the same session. After each session, participants rated the subjective activity of the music using a 101-point rating scale ranging from 0 (not active) to 100 (very active). The rating scale was created by the experimenter. We asked the participants to subjectively estimate how active they thought the four types of music were. This value indicated the degree to which the participants were stimulated by the four types of music. We hypothesized that the more stimulating and active the music was rated, the higher the arousal level of the participants would be, and that the perceived activity of the four types of music and the strength of their respective degrees of vection would be correlated. The sound level of the music ranged from $0 \mathrm{~dB}$ to $+20 \mathrm{~dB}$ for each condition. The average loudness of the four-minute piece of music was consistent. The sounds were emitted by a stereo-speaker (Computer MusicMonitor, BOUSE) (Figure 1). All participants participated in all five conditions (four music and one no music conditions). The order of conducting the five conditions was randomized.

\section{Result and Discussion}

The average latencies and durations were shortest and longest respectively in the two fast music conditions, shorter in the two slow music conditions, and slowest in the no-music condition (Figure 2). A one-way ANOVA revealed a significant main effect of the five conditions (latency, $F(4,52)=6.01, p$ $<.01$; duration, $F(4,52)=6.89, p<.01)$ ). Multiple comparisons revealed significant differences between the fast and slow music conditions, between the no-music and the two fast conditions, and between the no-music and Kanon-D-dur conditions, both in terms of latency and duration (Tukey's HSD, $p<.05$ ).

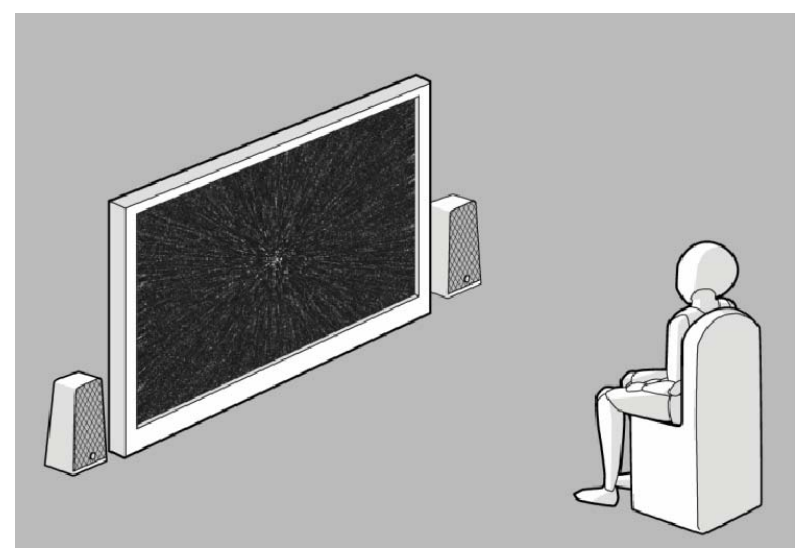

Figure 1.

A schematic illustration of the experimental environment.
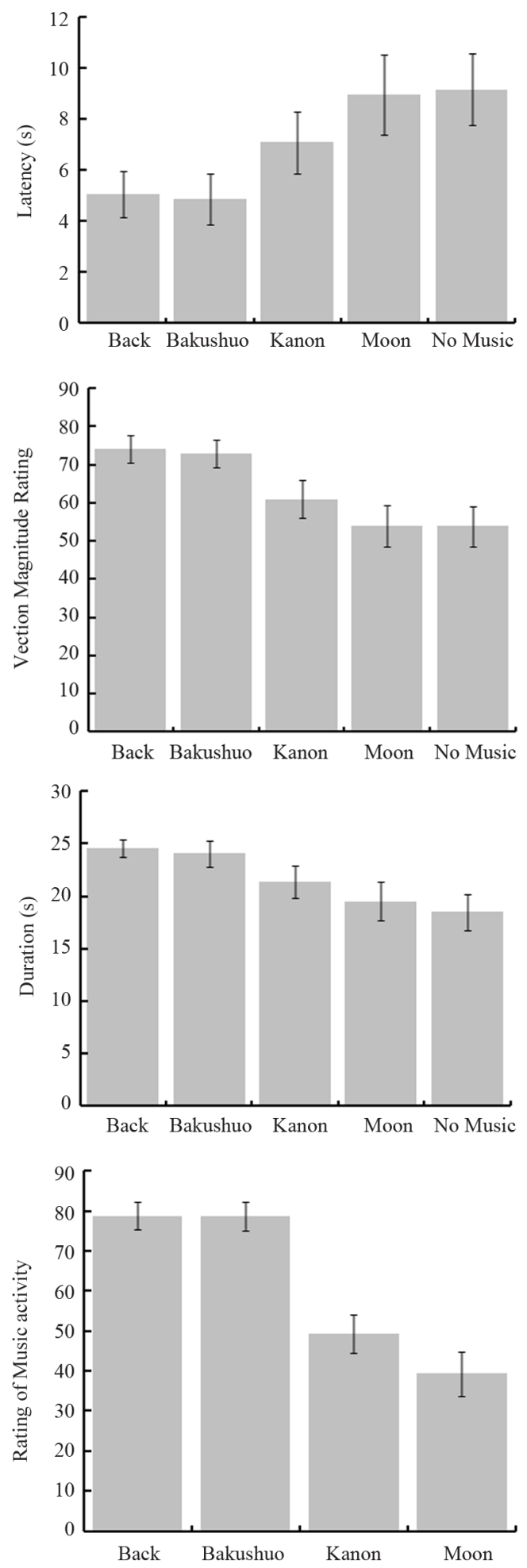

Figure 2.

Results of vection strength (latency, duration and magnitude) and subjective ratings of Music activity.

The average magnitudes were largest in the two fast music conditions and the Kanon-D-dur condition, and smallest in the Moon Light and no-music conditions. A one-way ANOVA revealed a significant main effect of the five conditions $(F(4,52)$ 
$=11.60, p<.01)$. Multiple comparisons revealed significant differences between the fast and slow music conditions, between the no-music and the two fast conditions, and between the no-music and Kanon-D-dur conditions (Tukey's HSD, $p$ $<.05$ ). The difference between the Kanon-D-dur and Moon Light conditions trended towards significance (Tukey's HSD, $p$ $<.1)$.

The average estimated subjective activity of the music was largest in the two fast music conditions and the Kanon-D-dur condition, and smallest in the Moon Light condition. A oneway ANOVA revealed a significant main effect of four conditions $(F(3,39)=56.35, p<.01)$. Multiple comparisons revealed significant differences between all combinations of these four conditions except between the two fast music conditions (Tukey's HSD, $p<.05$ ).

Here, we could speculate that the fast tempo music types stimulated the participants and that those music types induced higher arousal levels in the participants. The correlation between the subjective activity ratings of the music and the vection strength values implies that the music modulated arousal levels, which then modulated vection strength.

When participants listened to more active music, they perceived stronger vection. This result supports our hypothesis. The estimated subjective activity ratings of the music might indicate the depth of modulation of the arousal levels in the participants.

The presence of background fast tempo music had a facilitating effect on vection. Thus, we are able to present new empirical data related to how music can alter human perception. Our results suggest that music can alter human visual perception. Thus, visual illusions combined with musical stimuli, and the relationship between visual acuity and musical stimuli, should be examined in future work. The effects of combinations of visual and musical stimuli on human perception clearly constitute a new and promising topic for vision research.

We speculated that our results indicated that the induced higher arousal could induce stronger vection. Thus in the future by modulating participants' arousal level by presenting not only music but also some tactile stimuli and visual stimuli, vection strength can be modulated easily as intended directions.

In our study propose practical use of music to enhance visual effect, i.e. vection. In some virtual reality environment, vection should be enhanced by fast tempo music and also it can be modulated by the tempo of presence music. This can be a great advantage for the virtual reality contents developers.

\section{Acknowledgements}

I thank Tomomi Ogura for her beautiful figural design. The author was supported by Funds for the Development of Human Resources in Science and Technology (Japan Science and Technology Agency).This work is supported by Program to Disseminate Tenure Tracking System, MEXT, Japan.

\section{REFERENCES}

Brodsky, W. (2002). The effects of music tempo on simulated driving performance and vehicular control. Transportation Research, 4, 219-241.

Gabrielsson, A. (2001). Emotions in strong experiences with music. In P. N. Juslin, \& J. A. Sloboda (Eds.), Music and emotion: Theory and research (pp. 431-449). New York: Oxford University Press.

Herrington, J. D., \& Capella, L. M. (1996). Effects of music in service environments: A field study. The Journal of Services Marketing, 10, 26-41. doi:10.1108/08876049610114249

Husain, G., Thompson, W. F., \& Schellengerg, E. G. (2002). Effects of musical tempo and mode on arousal, mood, and spatial abilities. $\mathrm{Mu}$ sic Perception, 20, 151-171. doi:10.1525/mp.2002.20.2.151

Ihaya, K., Seno, T., \& Yamada, Y. (submitted) Più mosso: Fast self-motion accelerates mental tempo in virtual reality.

Iwamiya, S. (1997) Interaction between auditory and visual processing in car audio: Simulation experiment using video reproduction. Applied Human Science, 16, 115-119. doi:10.2114/jpa.16.115

Miell, D. E., MacDonald, R. A. R., \& Hargreaves, D. J. (2005). Musical communication. Oxford: Oxford University Press.

Mayfield, C., \& Moss, S. (1989). Effect of music tempo on task performance. Psychological Reports, 65, 1283-1290. doi:10.2466/pr0.1989.65.3f.1283

Milliman, R. (1982). Using background music to affect the behaviour of supermarket shoppers. Journal of Marketing, 46, 86-91. doi: $10.2307 / 1251706$

Milliman, R. (1986). The influence of background music on behaviour of restaurant patrons. Journal of Consumer Research, 13, 286-289. doi:10.1086/209068

Nittono, H., Tsuda, A., Akai, S., \& Nakajima, Y. (2000). Tempo of background sound and performance speed. Perception and Motor Skills, 90, 1122. doi:10.2466/pms.2000.90.3c.1122

Seno, T., Kawabe T., Ito. H., \& Sunaga, S. (2013) Vection modulates emotional valence of autobiographical episodic memories. Cognition, 126, 115-120. doi:10.1016/j.cognition.2012.08.009

Spinney, L. (1997) Pump down the volume. New Scientist, 155, 22. 